Ebisu Ebisu

Études japonaises Études japonaises

48 | automne-hiver 2012

Naissance d'une revue féministe au Japon : Seitō

(1911-1916)

\title{
Nora, Seitō, Xīn Qīngnián
}

Nora, Seitō, Xīn Qīngnián

$$
\text { ノラ・『青鞜』・『新青年』 }
$$

\section{Yōko Niimura}

Traducteur : Marion Razakariasa

\section{(2) OpenEdition}

\section{Journals}

Édition électronique

URL : http://journals.openedition.org/ebisu/592

DOI : 10.4000/ebisu.592

ISSN : 2189-1893

Éditeur :

Institut français de recherche sur le Japon (UMIFRE 19 MAEE-CNRS), Maison franco-japonaise

\section{Édition imprimée}

Date de publication : 1 septembre 2012

Pagination : $59-82$

ISSN : 1340-3656

\section{Référence électronique}

Yōko Niimura, « Nora, Seitō, Xīn Qīngnián », Ebisu [En ligne], 48 | automne-hiver 2012, mis en ligne le 21 mai 2014, consulté le 20 avril 2019. URL : http://journals.openedition.org/ebisu/592 ; DOI : 10.4000/ ebisu.592 


\title{
Nora, Seitō, Xīn Qīngnián
}

\author{
NIIMURA Yōko 新村容子*
}

Nous proposons dans cet article une étude comparative de la réception de Nora, personnage principal de la pièce du dramaturge norvégien Henrik Ibsen (1828-1906) intitulée Une Maison de poupée, au Japon et en Chine dans les décennies 1910 et 1920. Ibsen fut introduit au Japon pour la première fois en 1889 par Mori Ōgai 森鷗外 qui rentrait alors d'un voyage d'études en Allemagne. Lorsqu'en 1906 se répandit la nouvelle de la mort du dramaturge, le pays se prit de passion pour son œuvre (Nakamura 1997). Lu Xun 魯迅, qui étudiait à l'époque au Japon ${ }^{1}$, mentionna Ibsen dans un article publié en 1908. Il s'agit de la première introduction de l'auteur en Chine (Shimizu 1994 : 1). C'est donc par l'intermédiaire d'étudiants expatriés qu'Ibsen fut diffusé de l'Europe au Japon, puis du Japon à la Chine.

La fondation de Seitō 青鞜 eut lieu à Tokyo en septembre 1911, tandis que Xinn Qingnián 新青年 La Jeunesse (littéralement : Nouvelle Jeunesse) parut pour la première fois à Shanghai en septembre 1915. Seitō est connue pour être une revue de littérature féminine fondée par Hiratsuka Raichō 平塚らいてう qui souhaitait inciter les femmes à s'affranchir de la domination patriarcale, ainsi qu'à affirmer leur conscience en tant qu'individu et leur

* Université d'Okayama 岡山大学.

1. La victoire japonaise lors de la guerre sino-japonaise (1895), puis lors de la guerre russo-japonaise (1905), suscita auprès de la jeunesse chinoise un grand engouement pour les voyages d'étude au Japon (Gen 1991 ; Reynolds 1993). 
indépendance en tant qu'être humain. Xìn Qingnián est une revue à laquelle Hu Shi 胡適, rentré en 1917 d'un séjour d'études aux États-Unis, consacra toute son énergie afin d'inviter la jeunesse à se délivrer de la conception confucéenne de la famille, en opérant une réforme des consciences au profit de l'« individu ". Il apparaît donc que, de façon presque simultanée, des revues virent le jour dans les deux pays dans le but d'exhorter à une prise de conscience de l'« individu » et à l'indépendance en tant qu'être humain.

Ibsen retint à la fois l'attention de Seitō et de Xìn Qingnián. La " fugue ${ }^{2}$ " de Nora, héroïne d'Une Maison de poupée, devint en particulier l'objet d'un débat passionné. La revue Seitō publia en janvier 1912 un « Numéro spécial Nora ", tandis que Xìn Qingnián réalisa un " Numéro spécial Ibsen " en juin 1918. Nous nous attacherons en premier lieu à comparer les commentaires et les analyses sur Nora respectivement entrepris par Seitō et par Xìn Qingnián, pour dégager leurs similitudes et leurs divergences. Dans un second temps, nous réfléchirons à la manière dont les hommes japonais de l'époque réagirent face aux espoirs placés en Nora par les femmes de la revue Seitō. Enfin, nous examinerons dans un troisième temps quelle fut la réponse de la gent féminine chinoise à la position des intellectuels masculins qui prônaient l'abolition de la conception traditionnelle de la famille et l'union libre entre les deux sexes.

Il convient ici d'aborder la différence de statut social de la femme entre le Japon et la Chine d'alors. Contrairement à Seitō, dont la publication et la rédaction étaient entièrement prises en charge par des femmes, Xìn Qingnián était une revue dirigée par des hommes. Celle-ci contenait de nombreux essais traitant de la question des femmes, mais tous étaient écrits d'une main masculine. Dans la Chine des années 1910-1920, il était extrêmement rare qu'une femme se lance dans l'édition d'une revue ou devienne l'auteure d'un article ${ }^{3}$.

2. Le terme en japonais est iede 家出, fugue. Mais la décision de Nora de quitter le foyer ne peut vraiment pas être considérée comme une fugue. Nous gardons par commodité ce terme ici [N.D.E.].

3. Une femme chinoise déclarait en 1903 : « Confinée dans sa pièce, la Chinoise ne sait pas même qu'il existe un pays " (Sakamoto $2004: 1$ ). Néanmoins, un périodique intitulé Nǚxuébào 女学報 (Le Journal des femmes) fut fondé en octobre 1898, au moment fort de la réforme des Cent jours (Băirì wéixin 百日維新) initié par le jeune empereur Guangxu 光緒帝 et ses conseillers. Il s'agit d'une revue qui fait époque en raison de la 
La Chine et le Japon, tous deux de culture confucéenne, étaient des sociétés résolument patriarcales. Toutefois, sur le plan de l'éducation des filles, entre la fin du XVIII ${ }^{\mathrm{e}}$ et le début du XIX ${ }^{\mathrm{e}}$ siècle, le Japon n'était pas moins avancé que l'Europe. Jusque parmi les jeunes filles venues de la campagne pour occuper des emplois subordonnés dans les boutiques, il était courant de savoir lire, écrire et compter (Aoki 2000, 1993 : 210-218). On peut considérer que cet état de fait provenait davantage de l'attention accordée d'une manière générale à l'instruction du peuple que de la volonté d'éduquer les femmes, et que c'est par extension qu'elles bénéficièrent de cette faveur. À l'entrée dans l'ère Meiji, une importance accrue fut accordée à l'éducation nationale, et dès le début du $\mathrm{Xx}^{\mathrm{e}}$ siècle des écoles supérieures pour jeunes filles furent fondées ${ }^{4}$. Il est bien connu qu'au sein de la revue

composition féminine de son édition et de sa rédaction, mais sa parution s'interrompit après trois mois (Zhang 2006 : 94-99).

4. L'opposition entre la situation en Chine et au Japon à cette époque est à nuancer car, contrairement à l'idée couramment admise que la Nihon joshi daigakkō 日本女子 大学校 qu'intégra Raichō en 1903 était la première université pour jeunes filles, celle-ci n'avait pas le statut d'université. Au Japon à cette époque, aucune université n’admettait de femmes, ni en tant qu'étudiantes, ni en tant qu'auditrices libres. Yamakawa Kikue 山川菊栄 (1890-1980) raconte dans ses souvenirs l'amertume d'avoir essuyé un refus lorsqu'elle demanda à assister en tant qu'auditrice libre aux cours de psychologie de l'université impériale de Tokyo (Yamakawa 1956 : 136-137). Raichō évoque cette fermeture dans son "Manifeste ": " Je suis attristée de voir qu'au Japon où il n'existe qu'une seule université destinée aux femmes, les universités masculines ne montrent aucune générosité pour ouvrir leurs portes aux femmes ». La première à les avoir ouvertes est l'université impériale de Tōhoku en septembre 1913 (trois étudiantes sont admises), suivie par l'université impériale de Kyūshū (1925), puis de Hokkaidō (1930). L'université impériale de Tokyo n’accepta des auditrices pour la première fois qu'en 1920. Mais ni celle de Tokyo ni celle de Kyoto n’admirent des étudiantes régulièrement inscrites avant 1945. Dans le privé, l'université Waseda admit douze auditrices libres en 1921 et quatre étudiantes régulièrement inscrites en 1939. Avant 1900, il n'existe aucune école supérieure non plus pour jeunes filles, sinon l'École normale supérieure de Tokyo, ancêtre de l'actuelle université Ochanomizu, fondée en 1890 pour former les institutrices et enseignantes des écoles secondaires. Entre 1900 et 1901, trois établissements d'enseignement supérieur virent le jour : celui de Tsuda Umeko 津田梅子 (18641929), Joshi eigaku juku 女子英学塾 (Collège d'enseignement d'anglais pour jeunes filles), celui de Yoshioka Yayoi 吉岡彌生 (1871-1959), Tokyo jo.i gakkō 東京女医学校 (École de médecine pour jeunes filles de Tokyo) et enfin celui fondé par Yokoi Tamako 横井玉子 (1855-1903) et Satō Shizu 佐藤志津 (1851-1919), Joshi bijutsu daigakkō 
Seitō, nombreuses furent les diplômées de l'École d'enseignement supérieur pour jeunes filles (日本女子大学校 Nihon joshi daigakkō), à commencer par Hiratsuka Raichō (Horiba 1988) ${ }^{5}$.

En Chine, les femmes des familles de haut rang se virent octroyer une éducation en interne à partir du XVI ${ }^{\mathrm{e}}$ et du XVII ${ }^{\mathrm{e}}$ siècles, ce qui permit l'apparition de poétesses (Mann 1997, chap. 4). Cependant, celles qui recevaient une telle instruction étaient bien peu nombreuses, et on ne peut donc assimiler ces conditions à une éducation nationale. La longue tradition de l'éducation en vue de passer les examens impériaux, strictement réservée aux hommes, se maintenait. Un tel contexte ne permettait guère l'apparition d'auteures, encore moins de revues publiées uniquement par des femmes.

\section{Seitō et Nora}

Lors de la création de Seitō en septembre 1911, la Bungei kyōkai 文芸協会 (Société des arts littéraires) ${ }^{6}$ donnait la première représentation au Japon

女子美術大学校 (École supérieure des arts pour jeunes filles). La même année, Naruse Jinzō 成瀬仁蔵 (1858-1919) fonda la Nihon joshi daigakkō que par commodité les historiens nomment la première université pour jeunes filles. Mais en réalité, aucune de ces écoles d'enseignement supérieur n'obtint le statut d'université, car le gouvernement continuait à se montrer réticent à l'égard de l'enseignement universitaire pour les filles. En 1904, la Nihon joshi daigakkō ainsi que le collège universitaire fondé par Tsuda Umeko obtinrent le statut de senmon gakkō 専門学校 (école spécialisée) selon le décret promulgué en mars 1903, ce qui leur donnait en principe le statut d'une école à vocation professionnelle et un diplôme reconnu comme tel. L'École de médecine pour jeunes filles l'obtint seulement en 1912. Quelques autres établissements ayant ce statut furent créés à la fin Meiji, comme la Dōshisha joshi senmon gakkō 同志社女子専門学校. En ce qui concerne la Chine, nous pouvons remarquer que le phénomène suit grosso modo la même chronologie : l'université s'ouvrit pour la première fois aux femmes en 1920 , mais elles ne pouvaient encore prétendre qu'au statut d'auditrices, et non à celui d'étudiantes régulières (Chūgoku joseishi kenkyūkai 2004 : 68-69) [N.D.E.].

5. Par ailleurs, ainsi que le remarque Horiba (1988 : 20-21), les jeunes femmes qui prirent la tête des polémiques au sein de la revue Seitō n'étaient pas les collaboratrices issues de la Nihon joshi daigakkō, mais plutôt des jeunes femmes d'origine plus modeste. 6. Fondée en 1906 par Shimamura Hōgetsu (voir note 2 dans l'article de Christine Lévy dans ce même dossier) puis reformée en 1909 avec l'aide de l'écrivain et dramaturge 
du drame d'Ibsen intitulé Une Maison de poupée. La mise en scène était l'œuvre de Shimamura Hōgetsu 島村抱月, et le rôle principal était tenu par Matsui Sumako 松井須磨子 (1886-1919) ${ }^{7}$. Cette pièce connut un important retentissement, à tel point qu'elle fut portée sur la scène du Théâtre impérial au mois de novembre. La question de savoir ce qu'Ibsen a voulu dénoncer à travers Une Maison de poupée est en elle-même un grand sujet de débat, auquel nous ne contribuerons pas dans cet article. En effet, dans le Japon d'alors, Nora, qui abandonne son époux, ses enfants, et quitte son foyer dans le but de pourvoir par ses propres moyens à son épanouissement humain, suscita tout à la fois l'adhésion et la réprobation en tant que symbole de la "femme nouvelle» (atarashii onna 新しい女) ${ }^{8}$. La Maison de poupée était lue comme le récit de la fuite de Nora hors de son foyer.

En janvier 1912, la revue Seitō (vol. 2, nº 1) lui consacra un « Numéro spécial Nora », au sein duquel Ueno Yō 上野葉, Katō Midori 加藤綠, Ueda

Tsubouchi Shōyō 坪内道遥 (1859-1935), la Bungei kyōkai est une troupe de théâtre figurant parmi les premières à avoir créé au Japon des pièces occidentales. Après des débuts portant davantage sur le théâtre shakespearien, la troupe prend quelques années plus tard un nouveau départ qui l'amène à s'inscrire dans la vague du théâtre moderne (shingeki 新劇), à l'instar du Théâtre libre (Jiyū gekijō 自由劇場), un groupe initié en 1908 par l'auteur et metteur en scène Osanai Kaoru 小山内薰 (1881-1928). La Bungei kyōkai joue alors notamment Ibsen, Bernard Shaw et Hermann Sudermann. Elle est dissoute en 1913 du fait du scandale suscité par la relation entre Shimamura Hōgetsu et Matsui Sumako (voir infra) [N.D.T.].

7. Shimamura Hōgetsu (1871-1918), écrivain, poète, metteur en scène et critique littéraire, dirigea à partir de 1906 la revue Waseda bungaku 早稲田文学 (Littérature de Waseda). Son amante Matsui Sumako, de son vrai nom Kobayashi Masako 小林正子, est une célèbre actrice de l'ère Taishō qui s'illustra particulièrement dans les rôles de Nora et d'Ophélie (Shakespeare, Hamlet) au sein de la Bungei kyōkai. Lorsque la troupe cesse son activité en 1913, le couple crée le Geijutsuza 芸術座 (le Théâtre d'Art) et continue de donner plusieurs représentations au Théâtre impérial de Tokyo, où Matsui Sumako connaît toujours un grand succès, notamment dans son rôle de Katusha (Tolstoi, Résurrection). Shimamura Hōgetsu décède en 1918 de la grippe espagnole en pleine préparation de Carmen, rejoint dans la mort deux mois plus tard par sa compagne qui se suicide dans les locaux de leur salle de répétition, le soir de la première [N.D.T.].

8. Le point de vue consistant à apprécier Nora avant qu'elle ne quitte sa maison, mais à ne plus l'aimer après son départ, est récurrent dans la rubrique du Yomiuri shinbun 読 売新聞 intitulée " Femmes plaisantes et déplaisantes révélées par la littérature ", publiée entre le 27 avril et le 28 juin 1912. Il apparaitt que, dans le Japon d'alors, la Maison de poupée fut appréhendée avec pour seule ligne de mire la «fugue » de Nora. 
Kimiko 上田君子, Hiratsuka Raichō et Yasumochi Yoshiko 保持研子 développèrent chacune leur "thèse sur Nora ». Parmi elles, Ueno Yō apporta une contribution majeure avec son article intitulé « De la Maison de poupée à la question des femmes " (Ningyō no ie yori josei mondai e『人形の家』より 女性問題へ) ${ }^{9}$. Nous synthétiserons en trois points son propos. En premier

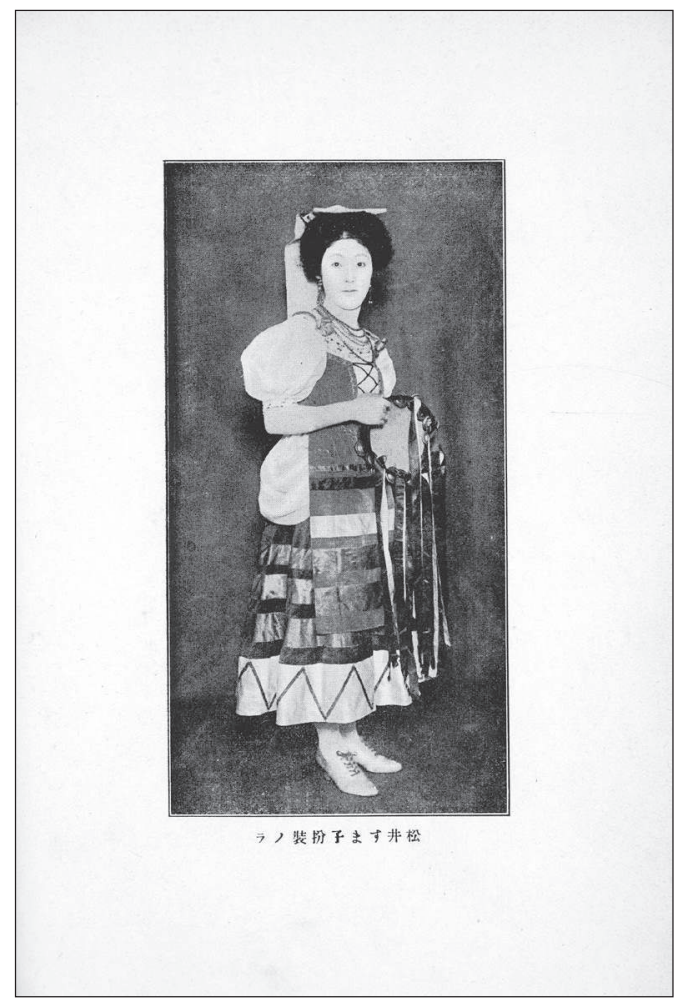

Fig. 1. Photo de l'actrice Matsui Sumako, publiée dans le numéro spécial de Seitō consacré à Nora, janvier 1912 (p. 60). Bibliothèque de l'université Waseda.

9. Seitō (vol. 2, n 1, janvier 1912 : 62-89) cité par Horiba (1991 : 34-46). Hasegawa Kei 長谷川啓 propose par ailleurs une présentation détaillée de l'article d’Ueno Yō (Hasegawa 1998). 
lieu, Ueno affirme que Nora s'est révoltée contre le système patriarcal. Elle " a dès à présent réalisé avoir subi un traitement indigne de la part de son père et de son époux ». Dans un deuxième temps, elle déclare que Nora fuyant son foyer " consacrera toute son énergie à la découverte de soi, à la culture de soi », et que seul relève de son urgent devoir l'affermissement de son individualité en tant qu'être humain à part entière. Enfin, elle soutient qu'il est grand temps pour la femme de se constituer une personnalité égale à celle de l'homme, et de susciter une révolution à l'encontre de ce dernier, de manière à permettre la transition entre le « régime despotique » régissant les rapports inhérents aux deux sexes et une " révolution " vers un « régime constitutionnel " prenant ses racines dans un respect et un amour mutuels. Il s'agit d'une doctrine virulente, exhortant à la révolution afin que les rapports entre les hommes et les femmes évoluent d'un "régime despotique " vers un "régime constitutionnel».

Le débat sur l'égalité de mode de vie avec l'homme traverse également l'article de Yasumochi Yoshiko intitulé "Au sujet de la Maison de poupée " (Ningyō no ie ni tsuite『人形の家』に就て) ${ }^{10}$. Celle-ci affirme qu’il n’y a pas lieu de distinguer un homme ou une femme par essence, " car ils ont la même valeur en tant que personne" (Horiba 1991 : 79), ajoutant que "la sottise de la femme est source de honte pour l'homme, et la sottise de l'homme source de honte pour la femme " (ibidem). Concernant le débat entre Ueno et Yasumochi, Hasegawa Kei évalue ainsi le caractère précurseur de leurs idées : "Elles soulèvent déjà une controverse dont les hommes eux-mêmes n'ont enfin compris la portée qu'aujourd'hui, dans la seconde moitié $\mathrm{du} \mathrm{Xx}^{\mathrm{e}}$ siècle, à savoir que la question des femmes est aussi celle des hommes " (Hasegawa 1998).

\section{La gent masculine japonaise et Nora}

Comment l'homme japonais de l'époque perçut-il la tentative de la part des femmes de la revue Seitō de susciter, ainsi que le présentait de manière symbolique Ueno Yō, une révolution dans les rapports entre les hommes et

10. Seitō (vol. 2, n $1: 143-154)$. Cet article est rédigé sous le nom de plume de "Y" (Horiba 1991 : 70-79). 
les femmes ? Dans le Japon d'alors, les femmes de la revue Seitō, assimilées à Nora fuyant son foyer, virent leur statut de femmes nouvelles devenir la cible des railleries et des moqueries. Les articles successifs publiés dans la rubrique intitulée "Femmes plaisantes et déplaisantes révélées par la littérature " ("Bungei ni arawaretaru suki na onna to kirai na onna "文 芸に現はれたる好きな女と嫌ひな女), entre le 27 avril et le 28 juin 1912 au sein du Yomiuri shinbun, dévoile clairement le sentiment des intellectuels japonais de l'époque. Le dramaturge et metteur en scène Matsui Shōyō 松居松葉 (1872-1933) y adressa le billet suivant ${ }^{11}$ :

"Les femmes qui me sont déplaisantes, au premier chef la Nora d'Ibsen, parlent de conscience de la femme et autres bien grandes choses, sans pourtant être encore capables d'appréhender tout à fait le monde, ni même de se confronter au terrain. Elles ne font que lutter contre des simulacres de tourments sans avoir vécu de réelles épreuves, c'est pourquoi, bien qu'elles recherchent le conflit avec leur mari, elles s'inventent des miracles et des échappatoires vers lesquels elles se précipitent. Si j'étais l'époux d'une telle énergumène, elle ne sortirait pas de la cuisine avant de s'être inclinée par trois côtés. "

Il s'agissait de reconnaître que là était la place dévolue à l'épouse au sein du foyer, et que s'opposer à son mari était un acte impardonnable.

Chiba Kikukō 千葉掬香 (1871-1938), un traducteur de métier ayant notamment pris en charge certaines œuvres d'Ibsen, rédigea lui aussi la note suivante ${ }^{12}$ :

"Elles ne sont certes point de celles que l'on nomme des femmes déplaisantes. Car c'est également cela qu'elles recherchent à toute force, ces prétendues Nora modernes, ces prétendues femmes conscientes."

Nul besoin de préciser que le néologisme de "prétendues Nora » désigne de façon sarcastique les femmes nouvelles.

Mais il se trouvait également parmi les hommes des personnes ayant bien accueilli la prise de conscience des femmes, et qui critiquèrent

11. Yomiuri shinbun, 19 mai 1912. Matsui Shōyō était le disciple de Tsubouchi Shōyō. Il eut également l'occasion de diriger la revue Waseda bungaku.

12. Yomiuri shinbun, 18 juin 1912. 
l'inconséquence des représentants de leur propre sexe. Suzuki 鈴木 livra ainsi cette longue contribution ${ }^{13}$ :

"Nora (Une Maison de poupée) - Magda (Le Pays natal) ${ }^{14}$. Ces femmes me sont toutes deux plaisantes. La plupart des Japonais sont aveuglément contraints au sacrifice de leur beauté. Ce qui nous est uniquement représenté comme une chose admirable. Dans le cas de la femme, c'est particulièrement démesuré. Puisqu'elle est déjà aveuglée, elle ne peut être pourvue ni d'une réelle valeur, ni d'une réelle beauté. Les deux femmes ci-dessus sont des personnes courageuses ayant envers et contre tout brisé le carcan de ces coutumes opprimantes. L'époux de Nora et le père de Magda ne sont-ils pas l'un comme l'autre de ces individus que l'on trouve en abondance dans le Japon actuel ? J'aimerais que l'ensemble des femmes de tout le pays me fassent la faveur d'éveiller leur conscience et, si leur conjoint est stupide (tel celui de Nora), qu'elles le laissent sans hésiter derrière elles en quittant leur foyer. Alors leur idiot d'époux ne pourra qu'en devenir plus humain. »

Les hommes embrassant des idées semblables à celles de Suzuki étaient néanmoins fort peu nombreux, la majorité d'entre eux semblant estimer, comme le romancier Tokuda Shūsei 徳田秋声 (1872-1943) ${ }^{15}$, que s'ils étaient en mesure d'admettre, sur le plan intellectuel, que la femme éveille sa conscience et devienne indépendante, s'agissant de la personne qu'ils choisiraient pour épouse, "en vérité cependant, c'est avec la femme classique, la femme captive que nous autres contemporains pouvons encore nous marier en toute quiétude ". Pour les hommes de l'époque, une femme désirable était de celles qui servaient leur époux et élevaient leurs enfants en

13. Yomiuri shinbun, 14 juin 1912. L'auteur est noté comme étant Suzuki Ei 鈴木鋭, mais l'on peut se demander s'il ne s'agirait pas en réalité de Suzuki Etsu 鈴木悦. Romancier et acteur du mouvement ouvrier, Suzuki Etsu (1886-1933) vécut une histoire d'amour avec Tamura Toshiko 田村俊子, déjà mariée à l'époque, et qu'il épousa plus tard. Également écrivaine, Tamura Toshiko contribua dès le premier numéro à la revue Seitō.

14. Magda (titre original : Heimat [Le Pays natal]) : pièce de l'écrivain et auteur dramatique allemand Hermann Sudermann (1857-1928) écrite en 1893 et mettant en scène la révolte de Magda, jeune femme indépendante et pleine d'assurance, contre l'autorité paternelle. Nora est une femme nouvelle en devenir, tandis que Magda est une femme nouvelle qui s'assume [N.D.T.].

15. Auteur très réputé au sein des cercles littéraires du mouvement naturaliste de l'époque. Son récit intitulé Shukuzu 縮図 (Miniature, 1941) figure parmi les œuvres emblématiques du roman moderne japonais. 
réprimant leur ego, capables de goûter « le charme du foyer - pesant, mais tout à la fois si doux » (Tokuda 1911: 50).

Tokuda reconnaissait pourtant que Nora, qui avait quitté son foyer, tout comme Hiratsuka Raichō et ses compagnes qui rejetaient le mariage conventionnel, « se devaient de s'atteler à la destruction des fondements du système familial hérité du passé et maintenu jusqu’à présent " (Tokuda 1911 : 51). La promotion de la doctrine de la «bonne épouse, mère avisée " participait alors de la formation d'un peuple japonais assujetti au régime impérial. Un défi de cette nature au système domestique revêtait de fait, aux yeux du pouvoir, un sens politique subversif. Pour cette raison, celui-ci tenta de faire disparaître de tels mouvements en répandant à leur sujet des calomnies et des attaques odieuses ${ }^{16}$.

Dans les années 1910, les femmes éveillées subissaient les violentes attaques du gouvernement et des médias de masse ${ }^{17}$, et se voyaient tournées en ridicule par les intellectuels du sexe opposé, mais ceux qui les faisaient le plus souffrir n'étaient-ils pas leurs partenaires masculins, totalement incapables de comprendre le sentiment de personnes dont le souhait était de construire un rapport d'égalité fondé sur l'amour ? Naganuma Chieko 長沼智恵子 (1886-1938), dans sa relation avec Takamura Kōtarō 高村光太郎 (1883-1956), sculpteur et poète, a elle-même cultivé et scellé son propre avenir de peintre et ses désirs de femme (Nakajima 2002) ${ }^{18}$. Cela n’a-t-il

16. Les autorités attaquaient la revue Seitō pour " atteinte à la vertu innée de la femme japonaise " et prirent souvent des mesures de censure à son encontre (Horiba 1988 : 168-169) ; Iwano Kiyo 岩野清, “Yasukōchi keiho kyokuchō no iken ni tsuite " 安河内 警保局長の意見について (Au sujet de l'opinion du chef du bureau de Police Yasukōchi) (Seitō, vol. 4, n 9 , septembre 1914 : 150-151), cité par Horiba (1991 : 214-217) ; Ueno Yō 上野葉, “Atarashii onna no tame ni : keiho kyokuchō no iken to iu o kikite " 新 しい女のために一警保局長の意見というをききて (Pour les femmes nouvelles. Après avoir entendu ce qui se trouve être l'opinion du chef du bureau de Police) (Seitō, vol. 4, n 9 , septembre 1914 : 152-158), cité par Horiba (1991 : 218-224).

17. Les médias de masse multipliaient les insultes abjectes visant la sexualité même des membres de la revue. Hiratsuka Raichō était accusée d'être anormale sur le plan sexuel et d'être hermaphrodite (Horiba 1988 : 174-176).

18. Naganuma Chieko figure parmi les rares femmes ayant appartenu au courant yōga 洋画, l'école de peinture japonaise de style occidental. Elle est notamment la conceptrice de la couverture du premier numéro de la revue Seitō. Son époux Takamura Kōtarō est aussi connu pour ses poèmes, en particulier ceux adressés à Chieko, Chieko shōo 智恵子抄 
pas échappé à son compagnon ? Quant à Ōsugi Sakae 大杉栄, qui traita en " amantes " Kamichika Ichiko 神近市子 et Itō Noe 伊藤野枝, il ne mérite pas qu'on s'y attarde ${ }^{19}$. Dans le Japon des années 1910, les femmes éveillées, parce qu'elles l'étaient, eurent à souffrir de leurs rapports avec des partenaires masculins conservant un point de vue archaïque sur la gent féminine.

\section{Xìn Qīngnián et Nora}

Contrairement à la revue Seitō, qui rassemble sous la direction de femmes des ouvres littéraires et des essais écrits par des femmes, Xìn Qingnián, revue fondée en 1915 par Chen Duxiu 陳独秀, ne compte parmi ses collaborateurs que des hommes, tous d'éminents intellectuels tels que Hu Shi, $\mathrm{Lu}$ Xun, Zhou Zuoren 周作人 ou encore Cai Yuanpei 蔡元培 ${ }^{20}$. Précurseur

(Sélection de poèmes sur Chieko), recueil publié trois ans après le décès de cette dernière en hôpital psychiatrique [N.D.T.].

19. Intellectuel anarchiste, Ōsugi Sakae (1885-1923) avait élaboré un " pacte " de bonne entente comportant trois clauses (l'indépendance économique, la résidence séparée et le respect de la liberté de l'autre) afin de pouvoir maintenir une triple relation amoureuse avec son épouse légale, Hori Yasuko 堀保子, et ses deux maîtresses Itō Noe et Kamichika Ichiko, accord devant également permettre à chacune de faire de même. Cependant, il était bientôt apparu que Hori Yasuko, du fait de son éducation, et Itō Noe, qui se trouvait alors dans une situation socialement réprouvée (elle venait de quitter son premier époux et son fils aîné en emportant son fils nouveau-né) n'étaient pas en mesure de pourvoir par elles-mêmes à leurs besoins économiques, tandis qu'Ōsugi en était venu à laisser paraître un réel amour pour Itō. Cette situation rendit caduque leur contrat et amena Kamichika, folle de rage, à blesser son amant au poignard durant ce que les médias nommèrent l'incident de Hikagejaya 日蔭茶屋. L'affaire défraya la chronique et jeta l'opprobre sur le couple Itō-Ōsugi, lesquels choisirent la vie commune à compter de cette époque, qu'ils maintinrent jusqu’à leur assassinat par la gendarmerie au lendemain du grand tremblement de terre du Kantō en septembre 1923 [N.D.T.]. 20. Chen Duxiu (1879-1942) est un homme politique qui participa à la fondation du Parti communiste chinois, dont il fut le premier secrétaire général de 1921 à 1927, avant d'en être évincé puis de rallier le mouvement trotskiste. Hu Shi (1891-1962), écrivain et philosophe, est notamment l'auteur d'un célèbre manifeste intitulé Wénxué găiliáng chíyi 文学改良㫚議 (Suggestions pour une réforme de la littérature), publié dans Xìn Qingnián en 1917 et défendant l'usage de la langue parlée dans la littérature (báihuà wèn 白話文). Il fut par la suite ambassadeur de la République de Chine aux États-Unis (1938-1942), puis président de l'université de Pékin (1946-1948). Lu Xun (1881-1936) 
en son genre, la revue exerça une influence prépondérante sur l'orientation idéologique du Mouvement du 4 mai $1919^{21}$. Elle diffère de Seitō du point de vue de la rédaction et des contributions, assumées par des hommes.

Xìn Qingnián bénéficiait d'un tirage de seize mille exemplaires, et sa popularité était telle qu'elle était épuisée dès sa parution, y compris en librairie. En particulier, le " Numéro spécial Ibsen » paru en juin 1918 semble avoir été très recherché (Shimizu 1995 : 2). Nous en extrayons ci-dessous la table des matières. Elle est constituée d'un essai de Hu Shi intitulé Yìbǔshēng zhǔyì 易卜生主義 (L'ibsénisme), de la traduction de trois pièces d'Ibsen, et de la biographie de ce dernier.

est l'auteur du premier récit écrit en langue parlée, Le Journal d'un fou (Kuángrén riji 狂人日記), paru dans Xīn Qingnián en 1918. Homme de lettres et professeur de littérature, Lu Xun s'investit également en politique, participant, entre autres, à la fondation de la Ligue des écrivains de gauche (1930) et de la Ligue chinoise des Droits de l'Homme (1933). Son frère Zhou Zuoren (1885-1967) est un essayiste qui soutenait une conception à la fois humaniste, démocratique et individualiste de la littérature. Il a été une des figures centrales du Mouvement du 4 mai 1919. Cai Yuanpei (18681940), après une brillante carrière au sein des plus hautes institutions éducatives impériales, démissionne de ses postes prestigieux afin de se consacrer à la politique ainsi qu'à l'étude des sciences humaines occidentales. Acquis aux idées libérales, il devient en 1916 président de l'université de Pékin qu'il entreprend de transformer en un établissement acteur du changement social, tout en respectant la liberté d'expression des différentes écoles de pensée, révolutionnaires comme conservatrices, présentes en son sein. Outre ces activités, il défend aussi le monde des arts en participant à la formation du futur Conservatoire de musique de Shanghai [N.D.T.].

21. Le Mouvement du 4 mai 1919, initié en grande partie par Xìn Qingnián, est un mouvement politique et culturel qui trouve son origine dans l'opposition, de la part de la jeunesse étudiante, à la légalisation de l'occupation japonaise de la province du Shandong (auparavant détenue par l'Allemagne) prévue par le Traité de Versailles au lendemain de la Première Guerre mondiale. Si la manifestation, qui rassembla près de trois mille étudiants devant la porte Tiananmen, connut par son ampleur des répercussions dans toute la Chine qui aboutirent au refus du gouvernement de signer le traité, le mouvement n'en perdit pas en vigueur pour autant, porté par une volonté de réforme culturelle et de transformation en profondeur de la société chinoise traditionnelle. Nombre de figures de proue de ce mouvement furent à l'origine de la fondation du Parti communiste chinois en 1921 [N.D.T.]. 
Yibǔshēng zhǔyì (L'ibsénisme) - Hu Shi

Une Maison de poupée

Premier acte - Luo Jialun 羅家倫

Deuxième acte - Luo Jialun

Troisième acte $-\mathrm{Hu}$ Shi

Un Ennemi du peuple - Tao Menghe 陶履恭

Le Petit Eyolf - Wu Ruo-Nan 呉弱男

Biographie d'Ibsen - Yuan Zhenying 袁振英

Nous nous intéresserons ici à l'article de Hu Shi. Celui-ci avait alors vingtsix ans et venait de rentrer d'un voyage d'études aux États-Unis achevé en 1917. La relation vécue là-bas avec Mademoiselle Williams (Edith Clifford Williams ${ }^{22}$ l'influença profondément sur le plan idéologique. La conviction selon laquelle la femme n'a pas à être traitée comme une poupée, mais qu'il y a lieu de la considérer comme un être humain à part entière, libre et autonome, lui fut acquise à travers sa fréquentation de la jeune Américaine. Shimizu Ken.ichirō soutient qu'il ne fait aucun doute que, pour $\mathrm{Hu}$ Shi, Mademoiselle Williams était Nora (Shimizu 1997 : 10-13).

En ouverture de Yibǔshèng zhǔyì, Hu Shi affirme que l'œuvre littéraire d'Ibsen appartient au courant réaliste, et qu'elle prend sa valeur dans sa fidèle description des divers aspects dépravés et abjects de la société, propos qu'il étend à la corruption de la société chinoise. Cette dernière position défendue par l'auteur vise à dénoncer les dessous du foyer. La famille chinoise est employée à l'oisiveté et au prestige de l'homme, et la polygamie ouvertement affichée. Sous couvert extérieur « d'humanité, de justice et de morale » (rényì dàodé 仁義道徳), le foyer en son sein n'est autre qu' "avidité et convoitise " (nándào nǘchāng 男盗女娼) ${ }^{23}$. Une institution de cette nature opprime l'esprit de liberté et d'indépendance de l'individu. Comment les jeunes devraient-ils vivre dans une telle société, semblable à un navire en perdition sombrant dans les ténèbres? Il incombe avant toute chose de se

22. Artiste du mouvement d'avant-garde new-yorkais, Edith Clifford Williams (18851971) étudie les beaux-arts à Yale (Yale School of the Fine Arts, New Haven), puis la peinture à Paris. Sa rencontre avec Hu Shi en 1914 marque le début d'une longue amitié ; leurs échanges épistolaires ne cessèrent qu’à la mort de ce dernier en 1962 [N.D.T.]. 23. Littéralement : "voleur et prostituée » [N.D.E.]. 
délivrer soi-même. Si Nora a quitté son foyer en y laissant son époux et ses enfants, c'est afin de se libérer, et bien que cela semble destructeur en apparence, il s'agit en réalité d'une action bel et bien constructrice. Hu Shi déclare ainsi que se délivrer soi-même et œuvrer au développement de son individualité propre sont les actes dont dépend le sauvetage d'une société qui présente tous les aspects d'un navire en naufrage.

Il existe de remarquables essais sur Lu Xun qui donnent la mesure de la contrainte et de la pression qu'exerça auprès des jeunes hommes, du $\mathrm{XVI}^{\mathrm{e}}$ au XIX ${ }^{\mathrm{e}}$ siècle, l'institution familiale chinoise - le clan - dont la principale fonction était alors la production d'hommes de l'élite admissibles aux examens impériaux (Maruyama 1965 ; Maruo 1993). À partir de 1905, ces épreuves mandarinales furent supprimées, ce qui conduisit le foyer à perdre sa caractéristique ; mais il est aisé d'imaginer la décadence et l'inertie d'un système familial incapable de se découvrir une nouvelle utilité. Dans

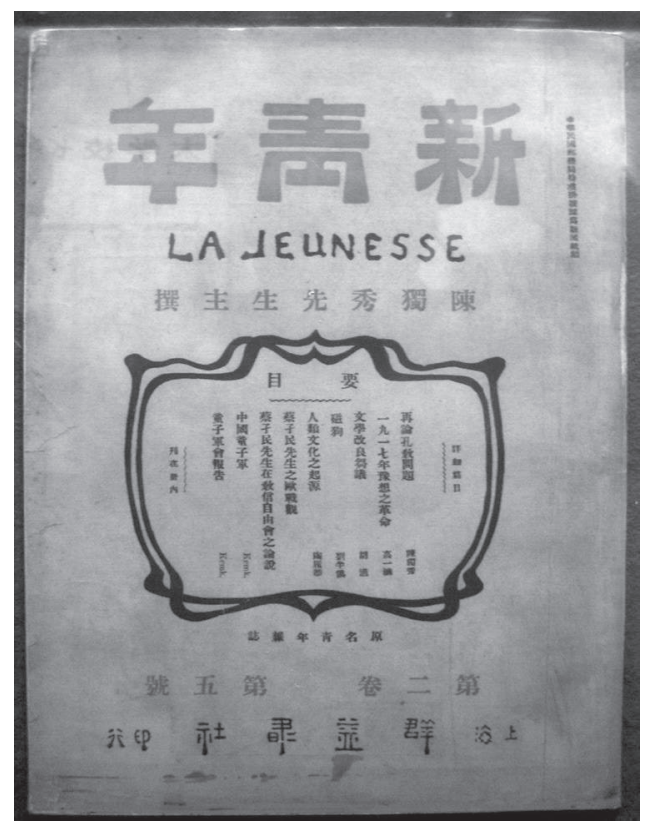

Fig. 2. Couverture de la revue Xīn Qingnián, fondée en 1915. 
Yỉǔshēng zhǔyì, Hu Shi encourage les jeunes à se libérer d'un foyer et d'une société plongés dans les ténèbres : s'émanciper soi-même pour sauver la société.

Faisant suite à son article publié en juin 1918, Hu Shi fait également paraître dans l'édition du mois de mars 1919 de Xìn Qingnián (vol. 6, n 3) un drame intitulé Zhöng shèn dà shì 終身大事. (La plus grande affaire de la vie). Inspirée d'Une Maison de poupée, la pièce est élaborée autour d'un scénario dans lequel l'héroïne, afin de réaliser son désir d'un mariage fondé sur l'amour libre, quitte la maison de ses parents, opposés à son union en raison de leurs idées surannées. Dans Zhōng shèn dà shì, la délivrance individuelle du joug de la société et de la famille, entités sombres et archaïques, est symbolisée par la réalisation d'un amour et d'un mariage libres grâce à la fuite du foyer parental.

\section{Une jeunesse en fugue}

Comme le dépeint de manière très vivante Shimizu Ken.ichirō, à cette époque, édifiés par la lecture de Yỉbǔshēng zhǔyì et de Zhōng shēn dà shì de $\mathrm{Hu}$ Shi, nombre de jeunes intellectuels s'enfuirent de leur foyer traditionnel et entreprirent de se mettre en quête d'une nouvelle voie. Ce furent les "Groupes solidaires de travail et d'étude ( Gōngdú hùzhù tuán工読互助団), formés entre fin 1919 et début 1920 à Beijing, qui procurèrent un lieu d'accueil aux fugitifs. Ces groupes étaient des associations d'étudiants proposant de mener de front étude et travail ; il s'agissait d'organisations révolutionnaires aspirant à la transformation de la société par le renversement de l'idée, fortement enracinée chez les intellectuels chinois classiques, selon laquelle le travail manuel serait une chose méprisable (Shimizu 1995 : 1).

Selon Shimizu Ken.ichirō, qui s'appuie sur les archives Hu Shi (húshi dàng’àn 胡適档案) récemment découvertes, ces «Groupes solidaires de travail et d'étude ", tout en jouant le rôle d'organisations révolutionnaires en vue d'une réforme radicale de la société, se trouvaient être, en outre, des lieux d'amours libres. Pour les jeunes intellectuels de l'époque, l'amour et le mariage libres constituaient un véritable pivot du changement social. Car « l'antique système familial était perçu par la jeunesse lettrée comme l'obstacle le plus important qui se dressait devant elle " (Shimizu 1995 : 3). 
Aspirant à la construction d'une nouvelle société dont ces deux idées seraient les symboles, les jeunes s'enfuirent de chez eux pour intégrer les "Groupes ». Au sein de la vie en commun qu’y expérimentèrent ces hommes et ces femmes (lesquelles n'étaient en revanche que peu nombreuses), la plus grande question à laquelle ils durent se confronter était également d'ordre passionnel : les querelles intestines impliquant les rapports entre les deux sexes étaient incessantes, et parfois mêmes sanglantes. Finalement, les "Groupes solidaires de travail et d'étude " échouèrent, principalement du fait des troubles internes occasionnés par ces relations (Shimizu 1995 : 10). Mais un tel échec a sans doute révélé, au contraire, combien l'amour libre et le mariage librement consenti constituaient un sujet vital pour la jeunesse d'alors.

\section{Les épouses traditionnelles délaissées}

L'écrasante majorité des jeunes qui participèrent à ces "Groupes " étaient des hommes. À cette époque, exceptée une infime minorité d'entre elles ayant eu l'occasion de recevoir une éducation, la plupart des femmes restaient confinées à leur domicile sans se voir offrir cette opportunité, et demeuraient même ignorantes des changements de la société. Entre la seconde moitié des années 1910 et les années 1920, le fossé séparant les jeunes intellectuels masculins, qui frayaient avec de nouveaux courants de pensée et s'éveillaient, à travers la revue Xìn Qingnián, à l'amour libre et au mariage librement consenti, des femmes n'ayant pas eu la possibilité d'entrer en contact avec ces nouvelles idées, était plus large que jamais.

Dans un article qui analyse "La Revue des femmes ", Fùnü zázhi 婦女雑誌 (publiée de 1915 à 1931), un périodique à destination de la gent féminine qui s'engagea en faveur du Mouvement du 4 mai, Xu Huiqi 許 慧琦 évoque comme suit la situation générale des jeunes hommes et des jeunes femmes de l'époque :

"Malgré le nom qu’arborait Fùnü zázhì, la majorité des postes, qưil s’agisse de la direction ou de l'équipe de rédaction, étaient occupés par des hommes. D'une manière générale, la femme ne possédait alors, comparativement à l'homme, qu'un pouvoir très réduit du point de vue de tout ce qui avait trait à l'écriture, à l'opinion publique, à la législation ou aux mœurs. De plus, il existait désormais un contraste irréconciliable entre les « filles traditionnelles " (surnom désignant à l'époque les femmes aux pieds 
bandés non alphabétisées) et les "garçons modernes " (les jeunes hommes ayant un savoir alphabétique élémentaire, côtoyant de nouveaux courants de pensée et possédant de surcroît une expérience d'études à l'étranger)» (Xu 2005 : 287).

La fracture dans le niveau de conscience entre les deux genres s'exprimait souvent, du côté des hommes, par des ruptures de fiançailles et des mouvements revendiquant la liberté de divorce. L'édition d'avril 1922 de Fùnü zázhi (vol. 8, n 4) consacra ainsi un numéro spécial au thème du divorce, et attribua plus de la moitié de ses pages à des courriers de lecteurs. Plus de quatre-vingt-dix pour cent de leurs auteurs étaient de jeunes hommes exprimant leur désir de rompre leur engagement et réclamant le « droit au divorce ». Ceci traduit l'importance du nombre de jeunes souhaitant mettre un terme à des fiançailles arrangées par leurs parents ou par des proches, sans tenir aucun compte de la volonté des personnes concernées (Xu 2005).

Xu Huiqi expose en ces termes les circonstances, publiées dans Fünü $z a ́ z h i$ (vol. 9, n 2), de la résolution de Zheng Zhenxun 鄭振壎, professeur à l'université du Sud-Est, de divorcer de son épouse " traditionnelle " ${ }^{24}$ :

"À l'âge de seize ans, alors qu'il était encore étudiant, Zheng Zhenxun se maria selon la décision de ses parents. Bien que le couple ait connu temporairement, dans les premiers temps de l'union, une douce lune de miel guidée en grande partie par le désir charnel et la passion spirituelle, les demandes répétées de la part de Zheng Zhenxun envers sa femme, telles que le fait de délier ses pieds bandés ou de cesser de se maquiller, ainsi que le refus catégorique de cette dernière, le conduisirent rapidement à se désintéresser d'elle. [...] Lorsqu'il en vint à enseigner à l'université de Beijing, il désira transformer son épouse en une femme nouvelle dotée d'un esprit d'indépendance, et la fit venir avec lui à Beijing, allant même jusqu'à lui confectionner le nouveau nom de Qiru 啓如 (" comme éveillée "). Mais tous ses efforts se soldèrent par un échec, ce qui le conduisit finalement à se résoudre au divorce » (Xu $2005: 289)$.

Le divorce de Zheng Zhenxun d'avec son épouse « traditionnelle " nous évoque également le cas de Lu Xun et de son épouse Zhu An 朱安.

Celle-ci avait été la fiancée choisie par la mère de Lu Xun alors que ce dernier était étudiant à l'École des chemins de fer et des mines, rattachée à l'École militaire du Jiangnan (janvier 1899 - janvier 1902). Lorsqu'il obtint son diplôme, Lu Xun poursuivit sur sa lancée en entamant un

24. L'article présenté est signé Kuàngfü 曂夫 (voir bibliographie). 
voyage d'études au Japon, qu'il poursuivit jusqu'en 1909. Cependant, en 1906, ayant reçu un télégramme l'informant que sa mère était mourante, il rentra chez lui provisoirement, pour y trouver accomplis tous les préparatifs d'une cérémonie de mariage. Lu Xun formula à plusieurs reprises envers Zhu An, à titre de contrat marital, les demandes de défaire ses pieds bandés et d'entrer à l'école, mais elle n'accepta ni l'une ni l'autre. Il la traita en étrangère, y compris après que leur union fut prononcée. Selon Takeuchi Minoru 竹内実, "du point de vue de Lu Xun, son mariage avec Zhu An était un inéluctable désastre, mais il en était certainement de même pour elle» (Takeuchi $2008: 212$ ).

Les requêtes de Zheng Zhenxun et de Lu Xun envers les épouses désignées pour eux par leurs parents se rejoignent de manière étonnante. Notamment en ce qui concerne l'abandon du bandage des pieds et l'acquisition d'une éducation en mesure de leur forger une personnalité indépendante. Zhu An ne savait vraisemblablement pas lire. Dans les deux cas, l'épouse rejeta la demande du mari. La différence réside dans le fait que Zheng Zhenxun prit la décision de divorcer, tandis que Lu Xun ne divorça pas, mais persista à se conduire en étranger avec sa conjointe, et se trouva une autre compagne. Il semble que Lu Xun était très conscient qu'en ce temps-là, une femme victime d'une rupture d'engagement ou répudiée n'avait nulle part où aller. Zhu An n'était certes pas traitée en épouse par Lu Xun, mais parce qu'elle travaillait pour la mère de l'écrivain, elle avait au moins trouvé un endroit où vivre.

Sans avoir connaissance du monde moderne, l'épouse de Zheng Zhenxun et Zhu An furent soudain sommées de changer ce qui constituait jusque-là leurs valeurs, cependant elles étaient certainement bien en peine de comprendre seulement ce que cela signifiait. Xu Huiqi fait la remarque suivante :

"Parce que les femmes ne se voyaient pas accorder l'occasion de recevoir une éducation ou de prendre un emploi, la grande vague des idées occidentales déferla sur la Chine sans qu'elles fussent dans la capacité d'accéder à ces nouveaux courants de pensée ni de les assimiler, et tandis que nombre de jeunes hommes se hasardaient à se laisser porter par la marée, l'écart entre les deux sexes allait croissant » (Xu 2005 : 290).

L'auteur soutient également que dans la mesure où ce n'étaient pas les femmes qui se trouvaient à l'initiative du mouvement d'émancipation féminine de l'époque, mais les hommes, celui-ci prit une direction tendant plutôt à ajouter, pour la convenance des hommes, une nouvelle oppression 
aux femmes (Xu 2003 : 138). Nous pouvons sans doute affirmer que les courants intellectuels nés dans les années 1920, qui prônaient l'indépendance de l'individu ainsi que son émancipation vis-à-vis d'un foyer et d'une société rongés par les vices, ont à l'inverse réservé un sort cruel à la femme chinoise.

\section{Conclusion}

À travers une étude comparative entre la revue Seitō, fondée par des femmes en 1911 au Japon, et la revue Xìn Qingnián, fondée en 1915 par de jeunes intellectuels chinois de sexe masculin, nous nous sommes intéressée au sein de cet article à la réception du drame d'Ibsen intitulé Une Maison de poupée, alors très en vogue dans les pays de l'Asie orientale. La revue Seitō publia en janvier 1912 un " Numéro spécial Nora ", et Xìn Qingnián un "Numéro spécial Ibsen » en juin 1918. Concentrant notre attention sur ces deux numéros spéciaux, nous avons étudié de quelle manière, à l'époque, fut perçue Nora, le personnage principal d'Une Maison de poupée, par l'intelligentsia féminine au Japon et l'intelligentsia masculine en Chine, tout en examinant les similarités et les divergences dans leurs réactions. Ajoutons que, si la Chine des années 1910 voyait enfin naître les premières revendications proclamant la nécessité d'assurer aux femmes une éducation ${ }^{25}$, la femme chinoise ne disposait pas d'un pouvoir suffisant pour publier des revues ou rédiger des articles. C'est pourquoi ce furent les hommes lettrés

25. Au Japon, c'est surtout au tournant du $\mathrm{xx}^{\mathrm{e}}$ siècle que la scolarisation des filles connaît un bond. Son taux avait très peu progressé durant les vingt-cinq premières années de Meiji, dépassant à peine les $50 \%$ en 1897, mais atteignit $96 \%$ en 1907. Les réticences de la part des familles avaient été très fortes à la campagne, où la fréquentation des écoles par les filles restait basse, de 36,5 \% en 1892, alors qu'elle atteignait 71,7 \% pour les garçons. Mais la diffusion de l'éducation s'est accompagnée d'une affirmation des rôles sexués au sein de la famille et de la société, avec des prescriptions sur le contenu des manuels, l'introduction de matières comme la couture pour les filles. L'État japonais avait décidé de faire face à l'influence des écoles fondées par les missionnaires - jugées délétères - en créant dans chaque département un enseignement du niveau de lycée pour les filles après avoir promulgué le Rescrit impérial sur l'Éducation en 1890 (Inoue 1975 : 134 sqq.) [N.D.E.]. 
qui soulevèrent en Chine les grandes thématiques de cette période, telles que la question des femmes ou celle de la famille.

Dans le numéro que la revue Seitō consacra à Nora, cette dernière fut dans l'ensemble favorablement accueillie. Ueno Yō, théoricienne reconnue, soutint en particulier qu'il était temps pour la femme de s'éveiller, à l'image de Nora, afin de se constituer une personnalité égale à celle de l'homme, et qu'il était nécessaire de transformer le "régime despotique " régissant les rapports entre les deux sexes en un "régime constitutionnel ».

D’une manière générale, au Japon, les revendications sérieuses ainsi exprimées dans la revue suscitèrent l'opposition de la part de l'intelligentsia masculine de l'époque. Elles donnèrent lieu à de véhémentes répliques objectant notamment qu'une femme n'avait pas à sortir de la cuisine, et qu'il était impardonnable de tenir tête à son époux. Les hommes de probité eux-mêmes, bien qu'ils fussent en mesure de comprendre l'égalité hommefemme sur le plan intellectuel, avouaient qu'en leur for intérieur, concernant la véritable vie de famille, ils gardaient une préférence pour la femme traditionnelle au service de son mari. En outre, nombre de membres de la revue Seitō eurent à souffrir de l'incompréhension de leur propre compagnon en la matière.

Du côté de la Chine, Nora fut perçue, d'une part, comme l'héroïne parvenue à s'enfuir d'une maison où lui étaient imposées les valeurs matrimoniales traditionnelles, afin de s'élancer vers l'univers nouveau de la liberté d'amour et d'hyménée. Les intellectuels chinois qui se trouvaient prisonniers d'un mariage résultant de l'accord entre deux familles entrevirent un espoir dans l'évasion de Nora, et les cas de jeunes gens fuyant leur domicile se multiplièrent. Blâmée au Japon par les hommes, à l'exception de rares féministes, pour l'abandon du foyer qu'elle impliquait, la « fugue » de Nora fut au contraire applaudie en Chine par la gent masculine.

D'autre part, exceptée une infime fraction de femmes, la majorité des Chinoises vivaient dans un monde auquel les notions d'amour et de mariage libres étaient étrangères. Elles n'avaient pas l'occasion d'être en contact avec les idées modernes de la revue Xìn Qingnián, et ne recevaient pas l'éducation qui leur aurait donné les moyens de les comprendre. Elles furent abandonnées par les intellectuels acquis à ces nouvelles conceptions.

Par la suite, quel fut donc le chemin que choisirent de suivre, l'une et l'autre, la femme japonaise et la femme chinoise ? La revue Seitō ne conserva son éclat que durant une brève période. Hiratsuka Raichō comme Yosano 
Akiko 与謝野晶子 (1878-1942), qui s'illustrèrent dans la polémique sur le " maternalisme» (bosei shugi 母性主義) qui les opposa l'une à l'autre, se rendirent captives du système impérialiste en endossant la fonction de chantres de la guerre (Ōmori 1997 : 168). Dans la société japonaise d'après 1945, la notion de partage des rôles selon les sexes, représentée par le soldat industriel d'un côté et l'épouse couvrant les arrières au foyer de l'autre, a persisté avec force, et subsiste encore de nos jours. Parmi ce que dénonçaient les femmes de la revue Seitō, lesquelles réalisèrent alors un grand pas en avant dans la redéfinition de ce que devrait être la nature de leurs rapports avec l'homme, il est encore des choses que, cent ans plus tard, nous commençons seulement à contester.

En ce qui concerne la Chine, le régime de guerre perdura longtemps à compter des années trente : quinze ans de combat contre le Japon impérialiste ; une guerre intérieure opposant nationalistes et communistes. Bien que la République populaire de Chine ait vu le jour en 1949, la situation demeura tendue avec les États-Unis et les pays de l'Union soviétique. Entre 1966 et 1976, la Grande Révolution culturelle fit sombrer le pays dans la guerre civile. Le régime de guerre se poursuivit pour ainsi dire jusqu'au début des années quatre-vingt. Dans ce long climat d'affrontement, on exigea de la femme qu'elle renie sa féminité et devienne un soldat, à l'instar de l'homme. La femme chinoise ne s'est-elle pas émancipée en prenant pour modèle le sexe opposé, sans faire l'expérience d'une évolution des mentalités émancipatrice de son individualité de femme ? Il apparaît ainsi que la conscience de sa propre richesse en tant que femme, comme la redéfinition de la nature des rapports avec l'homme ne cessent de constituer, y compris en Chine, un sujet des plus fondamentaux.

Traduit du japonais par Marion Razakariasa (université Paris Diderot-Paris 7) 


\section{Bibliographie}

AOKI Michio 青木美智男 1993

Taikei Nihon no rekishi. 11, Kindai no yochō 大系日本の歴史一11. 近代の予兆

(Abrégé de I'histoire du Japon. 11, Les signes de la modernité), Tokyo, Shōgakukan 小学館.

\section{AOKI Michio 2000}

" Kinsei kōki, dokusha to shite no Edo kasō shakai no josei : Shikitei Sanba Ukiyoburo o sozai ni » 近世後期、読者 としての江戸下層社会の女性一一式亭三馬 『浮世風呂』を素材に (La femme des couches sociales inférieures d'Edo comme lectrice dans la seconde moitié de l'époque moderne. D'après Ukiyoburo de Shikitei Sanba), Rekishi hyōron 歴史評論 (Critique historique), septembre, 605 : 37-41.

\section{Chūgoku joseishi kenkyūkai}

中国女性史研究会 2004

Chūgoku josei no hyakunen 中国女性の 100年 (Cent ans d'histoire de la femme chinoise), Tokyo, Aoki shoten 青木書店.

\section{Fùnü zázhì 婦女雑誌 (La Revue des} Femmes) 1915-1931

\section{GEN Ansei 厳安生 1991}

Nihon ryūgaku seishinshi : kindai Chūgoku chishikijin no kiseki 日本留学 精神史一一近代中国知識人の軌跡 (Histoire de l'esprit du voyage d'études au Japon : la trajectoire des intellectuels chinois de l'époque moderne), Tokyo, Iwanami shoten 岩波書店.
HASEGAWA Kei 長谷川啓 1998

" "Atarashii onna" no tankyū »〈新し い女〉の探求 (La quête des « Femmes nouvelles »), in Shin feminizumu hihyō no kai 新・フェミニズム批評の会 (Association pour une nouvelle critique féministe) (éd.), Seitō o yomu『青䩮』を 読む (Lire Seitō), Gakugei shorin 學藝書林, pp. 285-304.

\section{HORIBA Kiyoko 堀場清子 1988}

Seitō no jidai. Hiratsuka Raichō to atarashii onna tachi 青靱の時代 平塚らいてうと新しい女たち (L'époque de Seitō. Hiratsuka Raichō et les femmes nouvelles), Tokyo, Iwanami shoten 岩波書店.

\section{HORIBA Kiyoko 1991}

Seitō josei kaihō ronshū『青靼』女性解放 論集 (Recueil d'essais de la revue Seitō sur la libération de la femme), Tokyo, Iwanami shoten 岩波書店.

\section{IBSEN Henrik 1959}

Et Dukkehjem (Une Maison de poupée), traduction annotée de TAKEYAMA Michio 竹山道雄 (Ningyō no ie人形の家), Tokyo, Iwanami shoten 岩波書店.

IIDA Yūko 飯田祐子 (dir.) 2002

Seitō to iu ba. Bungaku, jendā, 《 atarashii onna »『青靱』という場—— 文学・ジェンダー・〈新しい女〉 (Seitō comme lieu. Littérature, genre, «femme nouvelle »), Tokyo, Shinwasha 森話社. 
INOUE Teruko 井上輝子 et alii 1975 Josei kaihō no shisō to kōdō 女性解放の 思想と行動 (L'émancipation des femmes : idées et actions), Tokyo, Jiji tsūshinsha 時事通信社.

\section{MANN Susan 1997}

Precious Records; Women in China's Long Eighteenth Century, Stanford Univ. Press.

\section{MARUO Tsuneki 丸尾常喜 1993}

Ro Jin. « Jin » « ki » no kattō 魯迅— 「人」鬼」の葛藤 (Lu Xun. Le conflit entre «l'homme » et «le monstre »), Tokyo, Iwanami shoten 岩波書店.

MARUYAMA Noboru 丸山昇 1965 Ro Jin. Sono bungaku to kakumei 魯迅一その文学と革命 (Lu Xun. Son œuvre et la révolution), Tokyo, Heibonsha 平凡社, coll. Tōyō bunko 東 洋文庫.

NAKAJIMA Miyuki 中島美幸 2002 "Shi to kaiga ni miru Seitō no joseizō» 詩と絵画に見る『青鞜』の性像 (La figure de la femme de Seitō à travers la poésie et la peinture), in IIDA Yūko 飯田祐子 (dir.), Seitō to iu ba. Bungaku, jendā, 《atarashii onna »『青鞜』という場— 文学・ジェンダー・〈新しい女〉 (Seitō comme lieu. Littérature, genre, « femme nouvelle»), Tokyo, Shinwasha 森話社.

NAKAMURA Toshiko 中村都史子 1997 Nihon no Ipusen genshō : 1906-1916 nen 日本のイプセン現象—1906〜1916 年 (Le phénomène Ibsen au Japon : 1906-1916), Fukuoka, Kyūshū daigaku shuppankai 九州大学出版会.
ŌMORI Kaoru 大森かほる 1997 Hiratsuka Raichō no hikari to kage 平塚らいてうの光と影 (Aspects clairs et obscurs de la vie de Hiratsuka Raichō), Tokyo, Daiichi shorin 第一書林.

\section{REYNOLDS Douglas R. 1993}

China, 1898-1912: The Xinzheng Revolution and Japan, Cambrige, Harvard University Press.

\section{SAKAMOTO Hiroko 坂元ひろ子 2004}

Chūgoku minzoku shugi no shinwa 中国民族主義の神話 (Le mythe du nationalisme chinois), Tokyo, Iwanami shoten 岩波書店.

\section{Seitōsha 青鞜社 1911-1916}

Seitō. Fukkoku-ban 青靼復刻版 (Seitō. Fac-similé), Tokyo, Ryūkei shosha 龍渓書舎, 1980 ; Fuji shuppan 不二出版, 1983.

\section{SHIMIZU Ken.ichirō 清水賢一郎 1994} «Kokka to shijin : Ro Jin to Meiji no Ipusen » 国家と詩人——魯迅と明治のイプ セン (L'État et le poète : Lu Xun et Ibsen à l'ère Meiji), Tōyō bunka 東洋文化 (Culture orientale), mars, $74:$ 1-33.

\section{SHIMIZU Ken.ichirō 1995}

«Kakumei to ren.ai no yūtopia : Ko Teki no "Ipusenshugi" to Kōdoku gojodan » 革命と恋愛のユートピア——胡適の〈イプ セン主義〉と工読互助団 (L'utopie de la révolution et de l'amour : «l'ibsénisme » de Hu Shi et les Groupes solidaires de travail et d'étude), Chūgoku kenkyū geppō 中国研究月報 (Le Mensuel des études chinoises), novembre, 49 (11) : 1-20. 
SHIMIZU Ken.ichirō 1997

« Nōra, jidōsha ni noru : Ko Teki Shūshin daiji o yomu »ノ一ラ、自動車に乗る—

胡適『終身大事』を読む (Nora en voiture : lecture de La plus grande affaire de la vie de Hu Shi), Tōyō bunka 東洋文化 (Culture orientale), mars, $77:$ 1-23.

\section{Shin feminizumu hihyō no kai} 新・フェミニズム批評の会 (Association pour une nouvelle critique féministe) (éd.) 1998

Seitō o yomu『青鞜』を読む (Lire Seitō)， Tokyo, Gakugei shorin 學藝書林.

TAKEUCHI Minoru 竹内実 2008 Kōrogi to kakumei no Chūgoku コオロギと 革命の中国 (La Chine du grillon et de la Révolution), Tokyo, Pī eichi pī kenkyūjo PHP研究所.

\section{TOKUDA Shūsei 徳田秋声 1911}

«Fujin no jikaku ni tsuite. Sono san » 婦人の自覚に就て一一其の三 (De l'éveil de la femme. Troisième partie), Waseda bungaku 早稲田文学 (Littérature de Waseda).

\section{Xīn Qingnián 新青年 La Jeunesse,} 1915-1922.

\section{XU Huiqi 2003}

«Nuola » zaì Zhōngguó : xín nǘxìng xíngxiàng de sùzaò jí qí yănbiàn, 1900s-1930s「娜拉」在中国: 新女性形象的 塑造及其演變、1900s-1930s (《Nora » en Chine : la fabrication et la transformation de l'image de la femme nouvelle dans les années 1900-1930), Taipei, Guoli zhengzhi daxue lishi xuexi 国立政治大学歴史学系.
XU Huiqi 許慧琦 2005

« Fujo zasshi kara miru jiyū rikon no shisō to sono jissen »『婦女雑誌』かみる 自由離婚の思想とその実践 (La pensée du droit au divorce et sa mise en pratique, vues à travers la Revue des Femmes), in MURATA Yūjirō (dir.) 村田雄二郎, Fujo zasshi kara miru kindai Chūgoku josei『婦女雑誌』らみる近代中国女性 (La femme chinoise moderne à travers la Revue des Femmes), Tokyo, Kenbun shuppan 研文出版, pp. 269-306.

Yomiuri shinbun 読売新聞 1912 « Bungei ni arawaretaru suki na onna to kirai na onna » 文芸に現はれたる好き な女と嫌ひな女 (Femmes plaisantes et déplaisantes révélées par la littérature), du 27 avril au 28 juin.

\section{ZHANG Lianbo 張蓮波 2006}

Zhōngguó jìndài fùnü jiěfàng sixxiăng lìchéng 中国近代婦女解放思想歴程 (Histoire intellectuelle de l'émancipation féminine dans la Chine moderne), Kaifeng, Henan daxue chubanshe 河南大学出版社.

\section{ZHENG Zhenxun 鄭振壎 (signé Kuàngfū} 曠夫 [Un homme célibataire]) 1923 《Wǒ zìji de hūnyīn shǐ » 我自己的婚姻史 (Histoire personnelle de mon mariage), Fùnü zázhì 婦女雑誌 (La Revue des Femmes), février, 9 (2) : 7-22. 